\title{
ON REPRESENTATION OF THE REEB GRAPH AS A SUB-COMPLEX OF MANIFOLD
}

\author{
MAREK KALUBA, WACŁAW MARZANTOWICZ*, AND NELSON SILVA ${ }^{\dagger}$
}

\begin{abstract}
The Reeb graph $\mathcal{R}(f)$ is one of the fundamental invariants of a smooth function $f: M \rightarrow \mathbb{R}$ with isolated critical points. It is defined as the quotient space $M / \sim$ of the closed manifold $M$ by a relation that depends on $f$. Here we construct a 1-dimensional complex $\Gamma(f)$ embedded into $M$ which is homotopy equivalent to $\mathcal{R}(f)$. As a consequence we show that for every function $f$ on a manifold with finite fundamental group, the Reeb graph of $f$ is a tree. If $\pi_{1}(M)$ is an abelian group, or more general, a discrete amenable group, then $\mathcal{R}(f)$ contains at most one loop. Finally we prove that the number of loops in the Reeb graph of every function on a surface $M_{g}$ is estimated from above by $g$, the genus of $M_{g}$.
\end{abstract}

\section{INTRODUCTION}

The Reeb graph $\mathcal{R}(f)$ of a function $f: M \rightarrow \mathbb{R}$ had been defined more than 60 years ago in [14] and [7], but just recently it has attracted more attention. It plays a fundamental role in computational topology for shape analysis $([2,4])$. Examples of applications of Reeb graphs include manifold reconstruction, 3D object indexing, $3 \mathrm{D}$ object recognition and many more. It is a very accessible invariant of the pair $(M, f)$ giving a simplification of the topological space $M$ and hence much desired from the computational point of view (cf. [5]). On the other hand, every graph which has no (oriented) cycles is represented as the Reeb graph of a $C^{1}$-function $f: M \rightarrow \mathbb{R}$ on a surface $M$, see $[11,16]$. This gives a converse relation from graphs to pairs $(M, f)$ and is done by two-dimensional surgery argument.

In this paper our aim is to provide a construction of the Reeb graph with a simplicial structure and as a subspace of $M$. We achieve it by constructing a finite one-dimensional complex $\Gamma(f) \subset M$ which is homotopy equivalent to $\mathcal{R}(f)$. The sub-complex is constructed via spaces of paths connecting critical points of $f$. The appropriate homotopy relation creates a bijective correspondence between the equivalence classes of paths and simplices of $\mathcal{R}(f)$.

Let us denote by $\pi:(M, f) \rightarrow \mathcal{R}(f)$ the canonical projection from the manifold to the Reeb graph, and let $\iota: \Gamma(f) \hookrightarrow M$ denote the inclusion. We prove that the composition

$$
\pi \circ \iota: \Gamma(f) \rightarrow \Gamma(f) \cong \mathcal{R}(f)
$$

induces the identity on the fundamental group of $\mathcal{R}(f)$ and this allows us to obtain a few relations between fundamental groups of $M$ and $\mathcal{R}(f)$. In particular we show

Date: September 6, 2021.

2010 Mathematics Subject Classification. Primary 57N65, 57R70; Secondary 57M50, 58K65.

Key words and phrases. Reeb graph, critical point, gradient flow.

* Supported by the Polish Research Grant NCN 2011/03/B/ST1/04533.

${ }^{\dagger}$ Supported by FAPESP of Brazil Grant BEPE 2012/15659-5. 
that if $M$ is simply-connected, or more general $\pi_{1}(M)$ is finite, then $\mathcal{R}(f)$ is a tree, i.e. a contractible finite graph. By the same argument we prove that if $\pi_{1}(M)$ is abelian, or more general if $\pi_{1}(M)$ does not contain $\mathbb{F}_{2}$, the free group on two generators, then $\mathcal{R}(f)$ contains at most one loop. This class of groups contains discrete amenable groups, thus nilpotent groups, solvable groups and many others (see [10]).

Finally, by a direct geometrical argument, we show that for a $C^{1}$-function $f: M \rightarrow$ $\mathbb{R}$ on a compact closed surface $M_{g}$ the number of loops of $\mathcal{R}(f)$ is less than or equal to $g$, the genus of $M$. This is a complementing result to that of [4] which says that for a Morse function $f: M \rightarrow \mathbb{R}$ the number of loops of $\mathcal{R}(f)$ is equal to $g$.

The paper is organised as follows. In Section 2 we set notation and global assumptions that hold throughout the paper. We also provide classic definitions and results obtained before, and prove some generalisations for $C^{1}$-functions. Section 3 is devoted to establish basic correspondences between sets of connected components, sets of homotopy classes of paths and edges and vertices of the Reeb graph. We join these results in Section 4, where we define and study $\Gamma(f)$. Finally, in Section 5 we apply these results to some questions concerning Reeb graphs on classical maifolds and we estimate number of loops in $\mathcal{R}(f)$ for a surface $M_{g}$.

\section{REEB GRAPH AND BASIC PROPERTIES}

Throughout the paper $M$ is a smooth (i.e. $C^{1}$ ) closed connected manifold of dimension $n \geqslant 2$ and $f: M \rightarrow \mathbb{R}$ is a $C^{1}$-function. Moreover we will assume that all critical points of $f$ are isolated.

Since $M$ is a compact space, the set of all critical points of $f$ (denoted by $\operatorname{Cr}(f) \subset M$ ) and the set of all critical values of $f$ (denoted by $\mathrm{V}_{c r}(f) \subset \mathbb{R}$ ) is finite. For a given $c, c^{\prime} \in \mathbb{R}$, we use the following notations:

$$
\begin{array}{ll}
M_{c}=f^{-1}(c), & M^{\left(c, c^{\prime}\right)}=\left\{x \in M: c<f(x)<c^{\prime}\right\}, \\
M^{c}=\{x \in M: f(x) \leqslant c\}, & M^{\left[c, c^{\prime}\right]}=\left\{x \in M: c \leqslant f(x) \leqslant c^{\prime}\right\} .
\end{array}
$$

As closed subsets, the sets $M_{c}, M^{c}, M^{\left[c, c^{\prime}\right]}$ are compact subspaces in $M$. The first set is called the level set of $f$. Note that

$$
M=\bigcup_{-\infty<c<\infty} M_{c}
$$

For a given $c \in \mathbb{R}$ let $M_{c}^{s}$ denote a connected component of the level set $M_{c}$. Now we recall the classical definition of the Reeb graph (see [7, 14]).

Definition 2.1. We say that points $x, y \in M$ are in the Reeb relation $x \sim y$, if $x$ and $y$ are in the same connected component $M_{c}^{s}$ of $M_{c}$. The quotient space $M / \sim$ is called the Reeb graph of $f$ and will be denoted by $\mathcal{R}(f)$.

The Reeb graph is a well defined entity by the virtue of the following lemma.

Lemma 2.2 (see $[7,14,16]$ ). The quotient space $\mathcal{R}(f)$ is homeomorphic to the body of a finite graph. Moreover, the vertices of $\mathcal{R}(f)$ correspond to the classes of $x \in$ $\operatorname{Cr}(f)$, i.e. to connected components $M_{c}^{s}$ such that $M_{c}^{s} \cap \operatorname{Cr}(f) \neq \varnothing$. Furthermore, $f$ induces a function $\tilde{f}: \mathcal{R}(f) \rightarrow \mathbb{R}$ such that $f=\tilde{f} \circ \pi$, which is strictly monotonic on each edge of $\mathcal{R}(f)$. 
Definition 2.3. Let $c \in V_{c r}(f) \subset \mathbb{R}$ be a critical value and let $M_{c}^{s}$ be a connected component of $M_{c}$. We call $M_{c}^{s}$ an essential component of $M_{c}$ if $M_{c}^{s} \cap \operatorname{Cr}(f) \neq \varnothing$. In other words, $M_{c}^{s}$ is essential if it contains a critical point. In the case $M_{c}^{s}$ is denoted by $M_{c}^{e s}$.

Proposition 2.4. Suppose that $M$ is a smooth compact manifold and that $f: M \rightarrow$ $\mathbb{R}$ is a $C^{1}$-function with isolated critical points. Let $c$ be a critical value and let

$$
A=\left\{x_{1}, x_{2}, \ldots, x_{n}\right\}=M_{c}^{e s} \cap \operatorname{Cr}(f)
$$

be the set of critical points in $M_{c}^{e s}$.

(1) Every path-connected component of a level set is its connected component.

(2) Every two different critical points $x_{1}, x_{2}$ in the closure of a connected component of $M_{c}^{e s} \backslash A$ can be connected by a path $\gamma: I \rightarrow M_{c}^{\text {es }}$ such that $\gamma(0)=x_{1}$, $\gamma(1)=x_{2}$, and $\gamma$ is a homeomorphic embedding (an arc).

(3) There exists a closed subspace $K \subset M_{c}^{e s}$ homeomorphic to a tree such that the set of vertices is equal to $A$.

Proof. Since a path-connected component is always a connected component, one inclusion of the first statement is obvious. For the other inclusion note that it is enough to study essential components of a level set. Otherwise, the level set is a manifold, hence path-connected components are its connected components.

Let $x_{0} \in M_{c}$ be a critical point and let $M_{c}^{s}=M_{c}^{e s}$ denote its connected component. It is enough to show that $M_{c}^{e s}$ is locally path-connected at each of its critical points.

Note that if $M_{c}^{e s}=\left\{x_{0}\right\}$ there is nothing to prove, so we may assume that $M_{c}^{e s} \neq$ $\left\{x_{0}\right\}$ and $M_{c}^{e s} \backslash\left\{x_{0}\right\}$ is a manifold locally near $x_{0}$. Indeed, for any $y \in M_{c}^{e s} \backslash\left\{x_{0}\right\}$ let $U_{y}$ be an open set in $M$ such that

- $U_{y}$ is diffeomorphic to an open disc in $\mathbb{R}^{n}$,

- $U_{y}$ does not contain any critical point of $f$,

- $U_{y}$ does not contain points of other connected component of $M_{c}$ besides $M_{c}^{e s}$.

Since $\left.f\right|_{U_{y}}: U_{y} \rightarrow \mathbb{R}$ is a submersion, $U_{y} \cap f^{-1}(c) \subset\left(M_{c}^{e s} \backslash\left\{x_{0}\right\}\right) \cap U_{y}$ is a submanifold.

Take a point $y \in M_{c}^{e s} \backslash\left\{x_{0}\right\}$ near $x_{0}$ and let $\widetilde{M}_{c}^{e s}\left(x_{0}, y\right)$ be a connected component of $M_{c}^{e s} \backslash\left\{x_{0}\right\}$ containing $y$. We claim that

$$
x_{0} \in \operatorname{cl}\left(\widetilde{M}_{c}^{e s}\left(x_{0}, y\right)\right),
$$

where $\operatorname{cl}(A)$ denotes the closure of $A$. Observe that $\widetilde{M}_{c}^{e s}\left(x_{0}, y\right)$ is open and closed in $M_{c}^{e s} \backslash\left\{x_{0}\right\}$. Suppose that $x_{0} \notin \operatorname{cl}\left(\widetilde{M}_{c}^{e s}\left(x_{0}, y\right)\right)$. Then $\operatorname{cl}\left(\widetilde{M}_{c}^{e s}\left(x_{0}, y\right)\right)=\widetilde{M}_{c}^{e s}\left(x_{0}, y\right)$ in $M_{c}^{e s}$, and $\widetilde{M}_{c}^{e s}\left(x_{0}, y\right)$ is open in $M_{c}^{e s}$, because $M_{c}^{e s} \backslash\left\{x_{0}\right\}$ is open. Therefore, $\widetilde{M}_{c}^{e s}\left(x_{0}, y\right)$ is a connected component of $M_{c}^{e s}$ which does not contain $x_{0}$. Since $M_{c}^{e s}$ is connected, $\widetilde{M}_{c}^{e s}\left(x_{0}, y\right)$ has to be empty, which is a contradiction.

Consider a descending family of open disks $\left\{D_{n}\right\}_{n \in \mathbb{N}} \subset M$ centred at $x_{0}$. We can assume that $y \in \partial \operatorname{cl}\left(D_{1}\right)$, the boundary of $\operatorname{cl}\left(C_{1}\right)$. Observe that $\partial \operatorname{cl}\left(D_{n}\right) \cap$ $\widetilde{M}_{c}^{e s}\left(x_{0}, y\right) \neq \varnothing$, for every $n$. Otherwise we would cover a connected set $\widetilde{M}_{c}^{e s}\left(x_{0}, y\right)$ by two open disjoint sets

$$
A=\widetilde{M}_{c}^{e s}\left(x_{0}, y\right) \cap D_{n} \quad \text { and } \quad B=\widetilde{M}_{c}^{e s}\left(x_{0}, y\right) \backslash D_{n} .
$$


Since $\widetilde{M}_{c}^{e s}\left(x_{0}, y\right)$ is locally a manifold it is path-connected. Set $x_{1}=y$. Let $x_{n} \in D_{n} \backslash D_{n+1}$ and $x_{n+1} \in D_{n+1} \backslash D_{n+2}$ be any points. Then for every $n$ there is a path $\gamma_{n}: I \rightarrow \widetilde{M}_{c}^{e s}\left(x_{0}, y\right)$ connecting $x_{n}$ and $x_{n+1}$.

We define a path $\gamma: I \rightarrow \widetilde{M}_{c}^{e s}\left(x_{0}, y\right) \cup\left\{x_{0}\right\}=M_{c}^{0}$ by the formula

$$
\gamma(t)= \begin{cases}\gamma_{1}(t) & \text { if } 0 \leqslant t \leqslant 1 / 2 \\ \gamma_{2}(t) & \text { if } 1 / 2 \leqslant t \leqslant 1 / 4 \\ \vdots & \vdots \\ \gamma_{n}(t) & \text { if } 1-1 / 2^{n} \leqslant t \leqslant 1-1 / 2^{n+1} \\ \vdots & \vdots \\ x_{0} & \text { if } t=1 .\end{cases}
$$

Consequently every point $y \in M_{c}^{e s}$ sufficiently close to $x_{0}$ can be connected to $x_{0}$ by a path. This shows that $M_{c}^{e s}$ is locally path-connected, thus path-connected. Note also that all the paths $\gamma_{n}$ used in the construction of $\gamma$ can be taken as homeomorphic embeddings of an interval (i.e. arcs). Consequently path $\gamma: I \rightarrow$ $M_{c}^{e s} \gamma(0)=y, \gamma(1)=x_{0}$ is a homeomorphic embedding of $I$, i.e. an arc, as the infinite composition of such arcs.

To prove the second statement we take two different critical points $x_{1}, x_{2} \in M_{c}^{e s}$. Let $y_{i}$ for $i=1,2$ be two points in a connected component of $M_{c}^{e s} \backslash \operatorname{Cr}(f)$ such that $y_{i}$ is in a neighbourhood $U_{i}$ of $x_{i}$. Moreover assume that $U_{1} \cap U_{2}=\varnothing$. Choosing a sufficiently small neighbourhood $U_{i}$ we may connect $x_{i}$ and $y_{i}$ by an arc $\gamma_{i}$ contained in $U_{i}$ for $i=1,2$. Since $y_{1}$ and $y_{2}$ are in the same component of $M_{c}^{e s} \backslash \operatorname{Cr}(f)$ we can find an arc $\widetilde{\gamma} \subset M_{c}^{e s} \backslash \operatorname{Cr}(f)$ connecting them. The composition of $\gamma_{1}, \widetilde{\gamma}$, and $\gamma_{2}^{-1}$ is an arc connecting $x_{1}$ and $x_{2}$. To ease the proof of the third statement denote this arc $\gamma$ by $\gamma_{2}^{1}$.

To prove the third statement we use a procedure similar to constructing a spanning tree. However, a special care is needed since we desire a geometric embedding of the tree in $M_{c}^{e s}$. In particular we require that edges are realised by $\operatorname{arcs}$ in $M_{c}^{e s}$ which do not intersect, except only in vertices.

Recall that $A=\left\{x_{1}, \ldots, x_{n}\right\}$ is the set of all critical points in $M_{c}^{e s}$. Fix $x_{1}$ as the starting point. Let $A^{1}=\left\{x_{1}^{1}, x_{2}^{1}, \ldots\right\} \subset A$ be the subset of $A$ containing points that can be connected with $x_{1}$ by arcs $\gamma_{j}^{1}$ as above such that $\gamma_{j}^{1} \cap \gamma_{j^{\prime}}^{1}=\left\{x_{1}\right\}$ for $j \neq j^{\prime}$. Set

$$
K^{1}=\bigcup_{x_{j} \in A^{1}} \gamma_{j}^{1}
$$

Then $K^{1}$ is homeomorphic to a one-dimensional complex contractible to $x_{1}$ (a hairy ball).

Apply consecutively the above construction to all $x_{j}^{1}$ in $A^{1}$ as the starting points. In each step connect $x_{j}^{1}$ analogously only to points in $A_{j}^{1}$, the set of these points in $A$ which have not appeared in the previous steps. We require that the joining arcs intersect only at $\left\{x_{j}^{1}\right\}$. Denote by $K_{j}^{2}$ such a one-dimensional complex contractible to $x_{j}$. Let

$$
K^{2}=K^{1} \cup \bigcup_{x_{j} \in A^{1}} K_{j}^{1},
$$


and observe that $K^{2}$ is homeomorphic to a tree rooted at $x_{1}$. Indeed, suppose that $K^{1} \cap K_{j}^{1} \neq \varnothing$, that is there is a cycle in $K^{2}$. This means that there are arcs $\gamma_{i}^{1}$ and $\gamma_{k}^{j}$ intersecting at a middle point $y$. Then

$$
\delta(t)= \begin{cases}\gamma_{i}^{1}(t) & \text { until } \gamma_{i}^{1}(t) \text { reaches } y \\ \gamma_{k}^{j}(t) & \text { afterwards }\end{cases}
$$

is an arc connecting $x_{1}$ to $x_{k}^{j}$. Therefore $x_{k}^{j}$ would belong to both $A^{1}$ and $A_{j}^{1}$ which is a contradiction. Repeating this construction we get a compact set $K \subset M_{c}^{e s}$ homeomorphic to a graph such that

- $\left\{x_{1}, \ldots, x_{n}\right\} \subset K$,

- all $x_{i}$ are vertices,

- $K$ is contractible to $x_{1}$.

which proves the proposition.

Corollary 2.5. Any two different critical points $x_{i}, x_{j} \in A$ can be joined by an arc which is a composition of arcs as above.

Remark 2.6. Given a (regular or critical) value $c \in \mathbb{R}$ of $f$, the topological space $M_{c}$ has a finite number of path-connected components $M_{c}^{s}$. The family $\left\{M_{c}^{s}\right\}$ forms an open cover of $M_{c}$ by disjoint sets, thus it is finite.

\section{Connected COMPONENTS AND ClAsses OF PATHS}

In this section we establish a correspondence between homotopy classes of paths and connected components of $M^{\left(c, c^{\prime}\right)}$. We start with well known facts about gradient trajectories in $M$. Then we prove that every connected component of type (IIa) (see Definition 3.6) corresponds bijectively to edges of $\mathcal{R}(f)$. Later we consider three increasing in generality families of paths: decreasing edge-paths, edge-paths and extended edge-paths (see definitions 3.16 and 3.21). On these families we introduce an equivalence relation - homotopy r.e.c. (see Definition 3.15) and prove that under the relation the families are the same.

At the end we establish a bijection between classes of homotopy r.e.c. of paths and connected components of $M^{\left(c, c^{\prime}\right)}$. The composition of bijections above allows us to identify edges (vertices) of $\mathcal{R}(f)$ and homotopy classes of extended edge-paths (contractible r.e.c. paths, respectively).

These results will be needed in Section 4 .

\subsection{Gradient trajectories.}

Definition 3.1. We say that a gradient flow-line $\gamma$ connects a critical point $x^{\prime} \in$ $M_{c^{\prime}}^{e s}$ to a point $x \in M_{c}^{e s}$ if

- $\lim \gamma(t)=x^{\prime}$ as $t \rightarrow-\infty$,

- if $x$ is a critical point then $x=\lim \gamma(t)$ as $t \rightarrow \infty$,

- if $x$ is a regular point there exists $t_{0}$ such that $\gamma\left(t_{0}\right)=x$.

Remark 3.2. To every trajectory as in the definition above, there corresponds a decreasing path denoted also by $\gamma: I \rightarrow M$, defined as follows. We take a monotonic smooth diffeomorphism from the real line (a closed ray, respectively) to the interval $(0,1)((0,1]$, respectively). The composition of the diffeomorphism with the trajectory map has a unique continuous extension to a path $\gamma:[0,1] \rightarrow \subset M$ such that $\gamma(0)=x^{\prime}, \gamma(1)=x$ in each of the cases above. 
The next proposition allows to effectively construct edges of graph $\Gamma(f)$ (to be defined in Section 4) as the integral curves of a differential equation. For an explanation of components of type (IIa) see Definition 3.6.

Proposition 3.3. Let $f: M \rightarrow \mathbb{R}$ be a $C^{1}$-function with finite number of critical points. Suppose that $C$ is a component of type (IIa) of $M^{\left(c, c^{\prime}\right)}$ such that $M_{c^{\prime}}^{e s}=$ $M_{c^{\prime}} \cap \operatorname{cl}(C)$. Then every point $x_{c^{\prime}} \in M_{c^{\prime}}^{e s} \cap \operatorname{cl}(C)$ can be connected with a point in $M_{c}^{e s}$ by the negative gradient trajectory $\gamma \subset \operatorname{cl}(C)$.

Proof. The proposition follows directly from the classical theorem stated below.

Theorem 3.4 (cf. [6, Chapter 1.6]). Let $M$ be a compact closed manifold equipped with a Riemannian structure, and $f$ be a $C^{1}$-function. Let $-\nabla f(x): M \rightarrow T M$ be the negative gradient vector field defined by $f$ and the Riemannian structure. Then the following conditions are satisfied.

(1) The field $-\nabla f$ is orthogonal to the level sets.

(2) The $\omega$-limit (positive) set $\omega_{-\nabla f}(x)$, and the $\alpha$-limit (negative) set $\alpha_{-\nabla f}(x)$ consist of critical points of $f$, i.e. are fixed points of the gradient flow.

(3) For any $x \in M$ and any $f$ the set $\omega_{-\nabla f}(x)$ is either a single point or an infinite set. The same holds for $\alpha_{-\nabla f}(x)$.

(4) If the function $f$ has only isolated critical points then every 1 non-trivial trajectory of $-\nabla f(x)$ converges to a critical point of $f$ as $t \rightarrow \pm \infty$.

Remark 3.5. Suppose that $\gamma$ connects $x^{\prime} \in M_{c^{\prime}}^{e s}$ to $x \in M_{c}^{e s}$. Even if $x^{\prime}$ is a critical point, $x$ may be a regular point. In particular, not every pair of critical points (in different level sets) are connected by a negative gradient trajectory (see [9, Chapter 2.3] for an example of a height function of a slightly inclined horizontal torus). For decreasing paths connecting critical points see Lemma 3.20.

\subsection{Connected components.}

Definition 3.6. Recall that $M^{\left(c, c^{\prime}\right)}$ was defined as $\left\{x \in M: f(x) \in\left(c, c^{\prime}\right)\right\}$.

- A connected component of $M^{\left(c, c^{\prime}\right)}$ is called of type (I) if it contains a critical point. Otherwise, it is called of type (II).

- Let $C$ be a component of type (II) of $M^{\left(c, c^{\prime}\right)}$. Suppose that

$$
f(\operatorname{cl}(C)) \cap \mathrm{V}_{c r}(f)=\left\{c, c^{\prime}\right\},
$$

i.e. $M_{c}^{e s} \cap \operatorname{cl}(C) \neq \varnothing$ and $M_{c^{\prime}}^{e s} \cap \operatorname{cl}(C) \neq \varnothing$. Then we call $C$ a component of type (IIa) or an edge-component.

In other words, component of type (IIa) is a connected component of $M^{\left(c, c^{\prime}\right)}$ which crosses every level set $M^{\left(c, c^{\prime}\right)}$ and its boundary intersects some essential components of $M_{c}$ and $M_{c^{\prime}}$.

Proposition 3.7. Let $C$ be a component of type (IIa) of $M^{\left(c, c^{\prime}\right)}$. There exists a diffeomorphism

$$
\left(M_{a} \cap C\right) \times\left(c, c^{\prime}\right) \cong M^{\left(c, c^{\prime}\right)} \cap C .
$$

Proof. Note that $g=\left.f\right|_{C}$ is a function without critical points. Consider the vector field $X=-\nabla g$. Since $\|X(p)\|>0$ for all $p \in C$, we can define $Y=\frac{X}{\|X\|}$ on $C$. In particular, it is defined on $g^{-1}([a, b])=M^{[a, b]} \cap C$ for any $a, b$ such that 
$c<a<b<c^{\prime}$. Let $\phi_{p}(t)$ denote the integral curve of $Y$ passing through $p \in g^{-1}(a)$. Since

$$
\frac{d \phi_{p}(t)}{d t}=1
$$

the flow of $Y$ which starts from the level $a$ at time 0 , will reach the level $b$ at time $b-a$. Therefore we obtain a diffeomorphism

$$
g^{-1}(a) \times[a, b] \ni(p, t) \longmapsto h(p, t)=\phi_{p}(t-a) \in g^{-1}([a, b]) .
$$

Now we want to extend the diffeomorphism to the whole connected component $C$.

Denote by $D$ the connected component $M_{d} \cap C$ of the level set $M_{d}$ for some $d \in\left(c, c^{\prime}\right)$. Consider an ascending family of intervals $\left\{\left[a_{n}, b_{n}\right]\right\}$, such that

$$
\bigcup_{n}\left[a_{n}, b_{n}\right]=\left(c, c^{\prime}\right) \quad \text { and } \quad d \in \bigcap_{n}\left[a_{n}, b_{n}\right] .
$$

Next we obtain a diffeomorphism

$$
h_{n}: D \times\left[a_{n}, b_{n}\right] \rightarrow g^{-1}\left(\left[a_{n}, b_{n}\right]\right)
$$

in a similar way as $h$ was obtained. By the uniqueness of the integral curve passing through a point, we can define a diffeomorphism as the direct limit

$$
\underset{n}{\lim _{n}} h_{n}: D \times \bigcup_{n}\left[a_{n}, b_{n}\right]=D \times\left(c, c^{\prime}\right) \longrightarrow \bigcup_{n} g^{-1}\left(\left[a_{n}, b_{n}\right]\right)=C
$$

by the formula

$$
(p, t) \longmapsto h_{n}(p, t) \quad \text { for some } n \text {. }
$$

Lemma 3.8. A component $C$ of type (II) of $M^{\left(c, c^{\prime}\right)}$ intersects exactly one connected component of $M_{d}$, for each level set $d \in\left(c, c^{\prime}\right)$.

Proof. Observe that $\left.f\right|_{C}: C \rightarrow \mathbb{R}$ does not have any critical point. Let $c<d<d^{\prime}<$ $c^{\prime}$. By the gradient flow argument (see Proposition 3.7) $M^{\left[d, d^{\prime}\right]}$ is homeomorphic to $I \times M_{d}$, and simultaneously to $I \times M_{d^{\prime}}$ which sets a bijection between connected components of $M^{\left[d, d^{\prime}\right]}$ and connected components of $M_{d}$ for all $d \in\left(c, c^{\prime}\right)$. Since $C$ intersects exactly one connected component of $M^{\left[d, d^{\prime}\right]}$, it intersects exactly one component of $M_{d}$.

Corollary 3.9. The manifold $M^{\left(c, c^{\prime}\right)}$ has a finite number of connected components.

Proof. The manifold $M^{\left(c, c^{\prime}\right)}$ decomposes as a union of type (I) and type (II) components. The number of critical points is finite, hence we have a finite number of components of type (I). Therefore it suffices to estimate the number of type (II) components.

Fix a regular value $d \in\left(c, c^{\prime}\right)$. Let $C$ be component of type (II). Note that $M_{d}$ has finite number of components as a compact sub-manifold of $M$. Since there are only finitely many of choices of $d$ which correspond to a different (non-canonically homeomorphic) level sets, the statement follows from the proof of Lemma 3.8.

Let us denote the interior of $J$ by $\int(J)$.

Proposition 3.10. There is a bijection between components of type (IIa) in $M$ and edges of $\mathcal{R}(f)$. 
Proof. The easy part is to show that $C$, a component of type (IIa) of $M^{\left(c, c^{\prime}\right)}$ satisfies

$$
\pi(C)=\int(J)
$$

for some 1-simplex $J \in \mathcal{R}(f)$. We just need to observe that

$$
\left[c, c^{\prime}\right]=f(\operatorname{cl}(C))=\tilde{f} \circ \pi(\operatorname{cl}(C))=\tilde{f}(J) .
$$

Therefore $\pi$ maps $\operatorname{cl}(C)$ bijectively to a set in $\mathcal{R}(f)$ satisfying:

- $\tilde{f} \circ \pi\left(\operatorname{cl}(C) \cap M_{c}\right)=c$,

- $\widetilde{f} \circ \pi\left(\operatorname{cl}(C) \cap M_{c}^{\prime}\right)=c^{\prime}$,

- no point in $\pi(C)$ is mapped via $\widetilde{f}$ to a critical value.

Since $C$ is connected, this is an edge of the Reeb graph.

Conversly, let $J$ be an edge in $\mathcal{R}(f)$ and let $x \in \pi^{-1}\left(\int(J)\right)$. Set $d=f(x)$ and consider $M_{d}(x)$, the connected component of the level set $M_{d}$ to which $x$ belongs. Since $f$ restricted to $\pi^{-1}\left(\int(J)\right)$ has no critical points, Lemma 3.8 implies that there exists a component $C_{i}$ of type (II) which contains $M_{d}(x)$. Define $C=\bigcup_{i} C_{i}$ to be the maximal (under inclusion) component satisfying these conditions. We claim that $\pi(C)=\int(J)$ and that $C$ is of type (IIa).

Observe that by definition $\pi\left(C_{i}\right) \subset \int(J)$. Suppose that there exists a point $y \in \int(J)$ such that $\pi^{-1}(y) \cap C=\varnothing$. Then using Proposition 3.7 we can show that $\pi^{-1}(y)$ is diffeomorphic to $\pi^{-1}(d)$. As $\pi^{-1}((y, d))$ is diffeomorphic to a cylinder, $C$ can be extended further to cover $\pi^{-1}(y)$. This contradicts the definition of $C$.

By the closed map lemma $\pi(\operatorname{cl}(C))=\operatorname{cl}(\pi(C))=J$, hence $C$ is of type (IIa).

Consider a relation defined on $M$,

$$
x \sim_{e s} y \in M \quad \text { if } \quad x, y \in M_{c}^{e s}, \text { for some } c \in \mathrm{V}_{c r}(f) .
$$

The relation induces an equivalence relation on $M$. We will denote the quotient space by $M_{e s}$. Let $\pi_{e s}: M \rightarrow M_{e s}$ be the canonical projection. Define $f_{e s}: M_{e s} \rightarrow$ $\mathbb{R}$ by $f_{e s}([x])=f(x)$. We have $f_{e s} \circ \pi_{e s}=f$ and the following lemma is a direct consequence of the definition.

Lemma 3.11. The Reeb graph of $f_{\text {es }}$, denoted by $\mathcal{R}_{\text {es }}(f)$ coincides with the Reeb graph of $f$.

Let $S(D)=D \times[-1,1] / \sim$ denote the suspension of $D$. Define a map

$$
\varphi: C \rightarrow S(D) \text { by } p \longmapsto\left[h^{-1}(p)\right],
$$

where $h$ is a diffeomorphism from Proposition 3.7. We can continuously extend the map to a map $\bar{\varphi}: \pi_{e s}(\operatorname{cl}(C)) \rightarrow S(D)$ by

$$
\bar{\varphi}(p)= \begin{cases}\varphi(p), & \text { if } p \in C, \\ {[x, 1],} & \text { if } p \in M_{c^{\prime}}, \\ {[x,-1],} & \text { if } p \in M_{c} .\end{cases}
$$

Since $\bar{\varphi}$ is continuous, bijective and its domain and image are compact and Hausdorff, $\bar{\varphi}$ is a homeomorphism. The proof of the following proposition is a straightforward consequence of Proposition 3.7. 
Proposition 3.12. Let $C$ be a component $C$ of type (IIa). The function

$$
\bar{\varphi}: \pi_{e s}(\operatorname{cl}(C)) \rightarrow S(D)
$$

is a homeomorphism.

Remark 3.13. Since $D$ is path-connected, $S(D)$ is simply-connected. Given a point $p \in D$ which is not in $\alpha$ - or $\omega$-set of a critical point, an embedded interval $\{p\} \times[a, b]$ is mapped to $\{p\} \times[-1,1]$ in $S(D)$, see Proposition 3.4. Contracting the interval yields $(\Sigma(D),[(y, 0)])$, the reduced suspension of $D$ which is a well pointed simplyconnected space.

Remark 3.14. It is worth of pointing out that our Proposition 3.12 provides a proof of the following theorem of Reeb.

Theorem. Let $M$ be a smooth manifold. Suppose that there exists a smooth function $f: M \rightarrow \mathbb{R}$ with exactly two non-degenerate critical points. Then $M$ is homeomorphic to the sphere $S^{m}$.

Indeed, in the situation the Reeb graph $\mathcal{R}(f)$ consists of just one edge connecting two vertices corresponding to the maximum and minimum (see also Lemma 5.8). Consequently $M=\pi_{e s}(\operatorname{cl}(C))$ is homeomorphic to the suspension of a level set $D$. Since critical points of $f$ are non-degenrated, it follows that $D$ is homeomorphic to a sphere $S^{m-1}$. The result follows. Note that without the assumption on nondegeneratedness, we cannot assume that $D$ is homeomorphic to a sphere. For example, degenerated maximum may have neighbourhoods homeomorphic to $C \Sigma$, the Brieskorn variaty (i.e. the cone on Brieskorn spheres).

In general, since $D$ and $S(D)$ are manifolds, it follows from the Poincare duality that $D$ is a $(m-1)$-homology sphere. Thus the suspension of $D$ is simply-connected homology sphere hence by Poincare conjecture it is homeomorphic to $S^{m}$.

\subsection{Families of paths.}

Definition 3.15. Let $c, c^{\prime}$ be two critical values of $f$ and let $\gamma_{1}, \gamma_{2}$ be two paths, such that

$$
\begin{aligned}
& f\left(\gamma_{1}(0)\right)=f\left(\gamma_{2}(0)\right)=c^{\prime} \quad\left(\text { that is } \gamma_{1}(0), \gamma_{2}(0) \in M_{c^{\prime}}^{e s}\right), \text { and } \\
& f\left(\gamma_{1}(1)\right)=f\left(\gamma_{2}(1)\right)=c \quad\left(\text { that is } \gamma_{1}(1), \gamma_{2}(1) \in M_{c}^{e s}\right) .
\end{aligned}
$$

- We say that a map $H: I \times I \rightarrow M$ is a homotopy relative to essential components (r.e.c.) between $\gamma_{1}$ and $\gamma_{2}$ when:

(1) $H$ is a homotopy between $\gamma_{1}$ and $\gamma_{2}$.

(2) $H(0, s) \in M_{c^{\prime}}^{e s}$ and $H(1, s) \in M_{c}^{e s}$, for all $s \in I$.

- A vertex-path is a path $\varepsilon: I \rightarrow M$ contained in an essential component of a critical level.

- We say that a path $\gamma$ is contractible r.e.c. if there exists a homotopy r.e.c. from $\gamma$ to a vertex-path.

Since level sets are path-connected, contraction r.e.c. is equivalent to the existence of a homotopy r.e.c. from $\gamma$ to a constant path $\sigma(t)=p \in \operatorname{Cr}(f)$, for some critical point $p \in M_{c}^{e s}$. Note that we can choose $p$ arbitrarily from the connected component of $M_{c}^{e s}$ to which $\gamma(0)$ belongs.

Definition 3.16. Let $C$ be a component of type (IIa) of $M^{\left(c, c^{\prime}\right)}$. An edge-path of $C$ is a path $\gamma$ such that 
- $\gamma(0) \in M_{c^{\prime}}^{e s}$ and $\gamma(1) \in M_{c}^{e s}$,

- $\gamma((0,1)) \subset C$.

We say that an edge-path is decreasing if

$$
f(\gamma(t))<f\left(\gamma\left(t^{\prime}\right)\right) \text { for } t>t^{\prime} .
$$

Lemma 3.17. Let $C$ be a component of type (IIa). Suppose that two edge-paths $\gamma_{0}$ and $\gamma_{1}$ are homotopic via homotopy $H$ fixing the endpoints in $\pi_{e s}(\operatorname{cl}(C)) \cong S(D)$. Then they are homotopic r.e.c. in $\operatorname{cl}(C)$.

Proof. Lifting of the homotopy in $\pi_{e s}(\operatorname{cl}(C))$ to $\operatorname{cl}(C)$ amounts to finding appropriate path in $\operatorname{cl}(C) \backslash C$.

For every step $s$ of $H(t, s)$, the homotopy from $\gamma_{0}(t)$ to $\gamma_{1}(t)$, let $x_{s} \in M_{c^{\prime}}^{e s}$ denote the limit

$$
x_{s}=\lim _{t \rightarrow 0} H(t, s)
$$

taken in $\operatorname{cl}(C)$. This is a valid definition since $C$ is diffeomorphic to $S(D)$ with the top point and the bottom point removed. By continuity of $H, \phi_{c^{\prime}}(s)=x_{s}$ is a path in $M_{c^{\prime}}^{e s}$. The homotopy r.e.c. in $\operatorname{cl}(C)$ from $\gamma_{0}$ to $\gamma_{1}$ can be obtained by pre-stacking $\left.H\right|_{C}$ with $\phi_{c^{\prime}}$ and post-stacking with (similarly defined) $\phi_{c}$.

Lemma 3.18. Let $\gamma$ be an edge-path of $C$. Then $\gamma$ is homotopic r.e.c. to a decreasing edge-path.

Proof. We will find a homotopy (fixing the endpoints) in $S(D)$ from $\pi_{e s}(\gamma)$ to a linear parametrisation of an interval connecting the top vertex and the bottom vertex. Then by Lemma 3.17 the proof is finished.

Let $\gamma$ be an edge-path of $C$ and set $D=M_{d} \cap C$, a connected component of a level set for some $d \in\left(c, c^{\prime}\right)$. Since $C$ is of type (IIa), $C$ is diffeomorphic (by Proposition 3.7) to $D \times(-1,1)$.

Choose a point $y \in D$ and a consider $\Pi: S(D) \rightarrow(\Sigma(D),[(y, 0)])$, the contraction of the interval $\{y\} \times[-1,1] \subset S(D)$. Observe that $(\Sigma(D),[(y, 0)])$ is the reduced suspension of $D$ which is a well pointed space. Under these contractions an edge-path $\gamma$ becomes $\Pi\left(\pi_{e s}(\gamma)\right)$, a loop based at $[(y, 0)]$. Since $D$ is connected, $(\Sigma(D),[(y, 0)])$ is simply-connected, hence $\Pi\left(\pi_{e s}(\gamma)\right)$ is contractible to $[(y, 0)]$.

To lift the contracting homotopy to $S(D)$ note that

$$
\Pi: S(D) \rightarrow(\Sigma(D),[(y, 0)])
$$

is a fibration such that the fibre has homotopy type of point (is a point or an interval to be precise). Since we can always lift the constant map $H(1, t) \equiv[(y, 0)]$, the homotopy lifting property provides a homotopy $H: I^{2} \rightarrow S(D)$ such that

$$
\left\{\begin{array} { l } 
{ H ( t , 0 ) = \pi _ { e s } ( \gamma ( t ) ) , } \\
{ H ( t , 1 ) \in \{ y \} \times [ - 1 , 1 ] , }
\end{array} \text { and } \left\{\begin{array}{l}
H(0, s) \in\{y\} \times[-1,1] \\
H(1, s) \in\{y\} \times[-1,1]
\end{array}\right.\right.
$$

Note that $\{y\} \times[-1,1]$ is a fibre over point $[(y, 0)]$. We want to modify $H$ to a homotopy $\widetilde{H}$ from $\pi_{e s}(\gamma)$ to a linear path

$$
\alpha(t)=(y,-1 \cdot t+1 \cdot(1-t))
$$

starting at $(y, 1)$ and ending at $(y,-1)$. 
Observe that $H(0, s)=\left(y, r_{0}(s)\right)$ and $H(1, s)=\left(y, r_{1}(s)\right)$ for some functions $r_{i}:[0,1] \rightarrow[-1,1]$. Let $h_{1}^{r}:[0,1] \rightarrow\{y\} \times[-1,1]$ be the path from $(y, 1)$ to $(y, r)$ given by

$$
h_{1}^{r}(t)=\left(y,(1-t) 1+t r_{0}\right) .
$$

Then $h_{1}^{r_{0}(s)}$ is a path which begins at $(y, 1)$ and ends at $\left(y, r_{0}(s)\right)=H(0, s)$. For each $s \in I$ pre-stack $H$ with $h_{1}^{r_{0}(s)}$. We remark that the collection of $\left\{h_{0}^{r(s)}\right\}_{s}$ forms a linear homotopy from the interval $\{y\} \times\left[r_{0}(0)=1,1\right]$ to $\{y\} \times\left[r_{0}(1), 1\right]$, which keeps the initial point $(y, 1)$ fixed. The same construction can be applied to obtain $h_{-1}^{r_{1}(s)}$, a path from $\left(y, r_{1}(0)=-1\right)$ to $\left(y, r_{1}(1)\right)$. Post-stack $H$ with the whole homotopy given by $\left\{\left(h_{-1}^{r_{1}(s)}\right)^{-1}\right\}_{s}$ (this is the reversed homotopy).

Observe that for a single $s$, the pre- and post-stacked $\left.H\right|_{\{s\}}$ becomes a path from $h_{1}^{r_{0}(s)}(0)=(y, 1)$ to $h_{-1}^{r_{1}(s)}(1)=(y,-1)$. Since we are stacking homotopies, the whole family of pre- and post-composed paths is continuous in $s$, and therefore is a homotopy

$$
\widetilde{H}: I^{2} \rightarrow S(D),
$$

from $\pi_{e s}(\gamma)$ to $\{y\} \times[-1,1]$ which keeps the endpoints fixed.

Corollary 3.19. Every two edge-paths of $C$ are homotopic r.e.c. .

Proof. By the above lemma, such edge-paths are homotopic to a decreasing edgepath given by an interval $[(y, t)]$ in $S(D)$. Since every such intervals are homotopic r.e.c. by a 'linear' homotopy (see proof of Lemma 3.20), the corollary follows by application of Lemma 3.17.

Lemma 3.20. Every decreasing edge-path of $C$ is homotopic r.e.c. to a decreasing edge-path of $C$ connecting two critical points.

In other words, every decreasing edge-path of $C$ is homotopic r.e.c. to a decreasing edge-path $\gamma$ of $C$ such that $\gamma(-1)=x_{c^{\prime}} \in M_{c^{\prime}}$ and $\gamma(1)=x_{c} \in M_{c}$ are critical points.

Proof. By virtue of Lemma 3.17 it is enough to prove the existence of such homotopy in $S(D)$.

Let $\gamma$ be a decreasing edge-path. From the argument in proof of Lemma 3.18 it follows that $\pi_{e s}(\gamma)$ is homotopic r.e.c. to a longitudinal path $\gamma_{0}:[-1,1] \rightarrow S(D)$ defined as $\gamma_{0}(t)=[(y, t)]$. Since $y \in D$ is arbitrary, we can assume that

$$
x_{c^{\prime}}=\lim _{t \rightarrow-1}(y, t) \in M_{c^{\prime}} \cap \operatorname{cl}(C)
$$

is a critical point by Proposition 3.3 (note that the limit is taken in $M$, and not in $S(D))$. By the same argument there exists a point $x \in D$ such that

$$
x_{c}=\lim _{t \rightarrow 1}(x, t) \in M_{c} \cap \operatorname{cl}(C) .
$$

In the following part of the proof we construct a 'linear' homotopy from $\gamma_{0}$ to (defined below) $\gamma_{1}$. Let $\omega$ be a path in $D$ such that $\omega(0)=y$ and $\omega(1)=x$. Furthermore, let $\phi(t)$ be a non-decreasing function $[-1,1] \rightarrow[0,1]$, such that $\phi(t) \equiv$ 0 on a small neighbourhood of $t=-1$ and $\phi \equiv 1$ near $t=1$. We define a path $\widetilde{\gamma_{1}}:[-1,1] \rightarrow S(D)$ by the formula

$$
\gamma_{1}=[(\omega(\phi(t)), t)]
$$


Note that $\gamma_{1}$ can be lifted to a (decreasing, by definition) path $\widetilde{\gamma_{1}}(t) \in \operatorname{cl}(C)$ such that

$$
\begin{gathered}
\widetilde{\gamma_{1}(-1)}=\lim _{t \rightarrow-1}(\omega(\phi(t)), t)=\lim _{t \rightarrow-1}(x, t)=x_{c^{\prime}}, \\
\widetilde{\gamma_{1}}(1)=\lim _{t \rightarrow 1}(\omega(\phi(t)), t)=\lim _{t \rightarrow 1}(y, t)=x_{c} .
\end{gathered}
$$

Finally, the formula

$$
\Lambda(t, s)=[(\omega(s t), t)]
$$

gives us a homotopy r.e.c. between $\gamma_{0}$ and $\gamma_{1}$. By Lemma 3.17, the initial path $\gamma$ is homotopic r.e.c. to $\widetilde{\gamma_{1}}$ what ends the proof.

Definition 3.21. Let $C$ be a component of type (IIa) of $M^{\left(c, c^{\prime}\right)}$. An extended edge-path of $C$ is a path $\gamma: I \rightarrow M$ such that:

(1) $\gamma(0) \in M_{c^{\prime}}^{e s}$ and $\gamma(1) \in M_{c}^{e s}$,

(2) There exist $t_{0}<t_{1} \in I$ such that

- if $f(\gamma(t))=c^{\prime}$ then $t \leqslant t_{0}$, and

- if $f(\gamma(t))=c$ then $t \geqslant t_{1}$, and

- $\gamma(t) \cap M_{d}^{e s}=\varnothing$, for $d \neq c, c^{\prime}$.

Lemma 3.22. Every extended edge-path $\gamma$ is homotopic r.e.c.to an edge-path of the form

$$
\gamma=\sigma * \alpha * \sigma^{\prime}
$$

where $\sigma^{\prime}, \sigma$ are vertex-paths contained in $M_{c^{\prime}}^{e s}, M_{c}^{e s}$ respectively, and $\alpha$ is a strictly decreasing edge-path.

Proof. Let $t^{\prime}$ be the largest $t_{0}$ such that the condition of the above definition holds. Then take $\widetilde{\sigma}^{\prime}$ to be (re-parameterized) $\left.\gamma\right|_{\left[0, t^{\prime}\right]}$. Similarly one may define $t^{\prime \prime}$ as the smallest $t_{1}$ and set $\widetilde{\sigma}=\left.\gamma\right|_{\left[t^{\prime \prime}, 1\right]}$. Finally, take $\widetilde{\alpha}=\left.\gamma\right|_{\left[t^{\prime}, t^{\prime \prime}\right]^{\prime}}$.

Observe that $\gamma$ is contained in $\mathrm{cl}(C)$. Moreover by condition (2) of the definition above $\widetilde{\sigma}^{\prime}$ intersects only $M_{c^{\prime}}$ and $\widetilde{\sigma}$ intersects only $M_{c}$. The image $\pi_{e s}\left(\widetilde{\sigma}^{\prime}\right)$ is a loop contained in a finite wedge of cones, hence is contractible to the middle point of this wedge. Therefore $\widetilde{\sigma}^{\prime}$ and $\widetilde{\sigma}$ are homotopic r.e.c. to $\sigma^{\prime}$ and $\sigma$, vertex-paths contained in $M_{c^{\prime}}$ and $M_{c}$ respectively.

Since all edge-paths are homotopic r.e.c. to decreasing edge-paths, the lemma is proved.

Corollary 3.23. Every extended edge-path of $C$ is homotopic r.e.c. to a decreasing edge-path.

Proof. By Lemma 3.22 an extended edge-path $\gamma$ could be written as $\sigma * \alpha * \sigma^{\prime}$, where $\sigma, \sigma^{\prime}$ are vertex-paths and $\alpha$ is an edge-path. Since vertex-paths are homotopic r.e.c. to constant paths, and every edge-path is homotopic r.e.c. to a decreasing one, lemma follows.

Theorem 3.24. Classes of homotopy r.e.c. of extended edge-paths are in bijection with edges of $\mathcal{R}(f)$. Moreover, each class of homotopy r.e.c. contains a decreasing path joining two critical points.

Proof. Given an extended edge-path $\gamma$ we may homotope r.e.c. it to appropriate edge-path by using Corollary 3.23. Since all edge-paths of $C$ are homotopic r.e.c. by Corollary 3.19 there is just one class of homotopy r.e.c. of extended edge-paths of 
C. By Lemma 3.20 we choose a decreasing representative which connects two critical points. Since component $C$ is of type (IIa) (by definition of edge-path), $C$ corresponds to a unique edge of $\mathcal{R}(f)$ by Proposition 3.10 .

\section{GraphS}

Let $V(f)$ be the set of all classes of homotopy r.e.c. of vertex-paths $[\varepsilon]$, and $E(f)$ be the set all classes of homotopy r.e.c. of extended edge-paths.

Definition 4.1. Let $\mathcal{G}(f)$ denote the set $\{V(f), E(f)\}$ and $V(f)(E(f))$ will be called the set of vertices (set of edges, respectively) of $\mathcal{G}(f)$.

We say that two vertices $\left[\varepsilon_{1}\right]$ and $\left[\varepsilon_{2}\right]$ are adjacent by edge if there exists an extended edge-path $\gamma: I \rightarrow M$ such that $[\gamma(0)]=\left[\varepsilon_{1}\right]$ and $[\gamma(1)]=\left[\varepsilon_{2}\right](\gamma(0)$ and $\gamma(1)$ are considered as constant maps). Note that there might be many different edges between two vertices.

Proposition 4.2. The pair $\mathcal{G}(f)=(V(f), E(f))$ defines a directed finite graph.

Proof. Given an extended edge-path $\gamma$ we consider the constant vertex-paths $\varepsilon_{1} \equiv$ $\gamma(0)$ and $\varepsilon_{2} \equiv \gamma(1)$. If $\gamma^{\prime} \in[\gamma]$, then paths $\varepsilon_{1}^{\prime} \equiv \gamma^{\prime}(0)$ and $\varepsilon_{2}^{\prime} \equiv \gamma^{\prime}(1)$ are homotopic r.e.c. to $\varepsilon_{1}, \varepsilon_{2}$, respectively. We associate the class $[\gamma]$ with the pair of vertices (a 1-simplex) $([\gamma(0)],[\gamma(1)])$. This endows $\mathcal{G}(f)$ with a simplicial structure.

By Corollary 3.9 we have a finite number of components of type (IIa), hence by Proposition 3.10 we have a finite number of edges. In every class of homotopy r.e.c. we may find a decreasing path $\gamma$ by Corollary 3.23 and we orient the edge in $\mathcal{G}(f)$ accordingly.

We write $[\gamma]=([\gamma(0)],[\gamma(1)], C)$ to indicate $C$, the component of type (IIa), to which $\gamma$ belongs.

Theorem 4.3. The graph $\mathcal{G}(f)$ is homeomorphic by a simplicial homeomorphism to $\mathcal{R}(f)$, the Reeb graph of $f$.

Proof. Consider the map $\mathcal{G}(f) \rightarrow \mathcal{R}(f)$ defined by

$$
\begin{aligned}
V(f) \ni[\varepsilon] & \mapsto \pi(\varepsilon(0)), \\
E(f) \ni[\gamma]=([\gamma(0)],[\gamma(1)], C) & \mapsto \pi(\operatorname{cl}(C)) .
\end{aligned}
$$

On vertices the map is bijective by the definition of the Reeb graph. By Theorem 3.24 , classes of extended edge-paths correspond bijectively to connected components of type (IIa). By Proposition 3.10, these components correspond bijectively to edges of the Reeb graph.

4.1. A realization of the graph $\Gamma(f)$ as a subspace of $M$. In this section we construct a finite one-dimensional complex $\Gamma(f) \subset M$ which is homotopy equivalent to $\mathcal{R}(f)$. For each critical value $c$ and for each component $C$ of type (IIa) choose a point $x_{c}^{C}$ such that $x_{c}^{C} \in \operatorname{cl}(C) \cap M_{c}^{e s}$.

Definition 4.4. We will denote by $\Gamma(f)$ the 1-dimensional CW-complex, defined as follows.

i) 0-cells are critical points of $f$;

ii) 1-cells are edges of a 'spanning tree' $\Gamma_{c}^{e s} \subset M_{c}^{e s}$ (provided by part (3) of Proposition 2.4), for every essential component in every level set. Moreover, 
iii) as additional 1-cells, in every component $C$ of type (IIa) we choose a decreasing edge-path $\gamma_{C} \in E(f)$ joining the chosen critical points. The existence of such $\gamma_{C}$ is proved in Lemma 3.20.

Proposition 4.5. Consider the restricted function $\left.f\right|_{\Gamma}: \Gamma(f) \longrightarrow \mathbb{R}$. Then there is a simplicial homeomorphism of the Reeb graph $\mathcal{R}_{\left.f\right|_{\Gamma}}$ of $\left.f\right|_{\Gamma}$ and $\mathcal{R}(f)$ which is orientation preserving.

Proof. Every point in $\Gamma(f)$ belongs either to the image of some $\gamma_{C}$, or to an essential component of a level set. Hence every point in $\mathcal{R}_{\left.f\right|_{\Gamma}}$ is either a single point from the image of $\gamma_{C}$, or an image of the whole $\Gamma_{c}^{e s}$. Define $\psi: \mathcal{R}_{\left.f\right|_{\Gamma}} \rightarrow \mathcal{G}(f)$ by

$$
\psi(p)= \begin{cases}t\left[x_{c}^{C}\right]+(1-t)\left[x_{c^{\prime}}^{C}\right] & \text { if } p=\pi\left(\gamma_{C}(t)\right) \text { for some } t \in(0,1), \\ {\left[x_{c}^{C}\right]} & \text { if } p=\pi\left(\Gamma_{c}^{e s} \cap C\right) .\end{cases}
$$

Recall that $\left[x_{c}^{C}\right]$ denotes the class of homotopy r.e.c. of a constant path. Since we chose exactly one representative $\gamma_{C}$ from every $C$, and $\gamma_{C}$ is decreasing, $\psi$ is linear on edges. Moreover $\psi$ is bijective on vertices, hence the composition

$$
\mathcal{R}_{\left.f\right|_{\Gamma}} \stackrel{\psi}{\rightarrow} \mathcal{G}(f) \rightarrow \mathcal{R}(f)
$$

is the required simplicial homeomorphism (the second map is given by Theorem 4.3). Finally orientation of $\mathcal{R}(f)$ is given by $f=f / \sim$ and corresponds to the orientation of $\mathcal{R}_{\left.f\right|_{\Gamma}}$ induced by $\left.f\right|_{\Gamma}$, thus these two orientations agree.

Remark 4.6. As a matter of fact we have

$$
\left.f\right|_{\Gamma / \sim}=f / \sim
$$

which induces the function

$$
\tilde{f}: \mathcal{R}(f) \cong \mathcal{R}_{\left.f\right|_{\Gamma}} \rightarrow \mathbb{R}
$$

Corollary 4.7. The graph $\Gamma(f) \subset M$ is homotopy equivalent to the Reeb graph $\mathcal{R}(f)$.

Proof. Note that the pre-image in $\Gamma(f) \rightarrow \mathcal{R}_{\left.f\right|_{\Gamma}}$ of every point is either a single point or a spanning tree $\Gamma_{c}^{e s}$. The result follows easily.

\section{Applications}

Let $\iota: \Gamma(f) \hookrightarrow M$ be the embedding given by Definition 4.4. The composition of maps

$$
\pi \circ \iota: \Gamma(f) \rightarrow \mathcal{R}(f)
$$

induces an isomorphism of the values of any homotopy functor, in particular of the homotopy and homology groups. Recall that $\pi_{1}(\Gamma(f)) \cong \pi_{1}(\mathcal{R}(f))$ which is a free group $\mathbb{F}_{r}$ on $r \geqslant 0$ generators.

Moreover, we can work in the pointed category, since a choice of a vertex $x_{0}$ of $\Gamma(f)$ as the distinguished point descends to a unique vertex $\pi\left(x_{0}\right)$ of $\mathcal{R}(f)$ which we set as the distinguished point of the latter. Consequently the first homology group $H_{1}(\mathcal{R}(f) ; \mathbb{Z})=\pi_{1}(\mathcal{R}(f))_{a b}=\mathbb{Z}^{r}$, and by Universal Coefficient Theorem $H^{1}(\mathcal{R}(f) ; \mathbb{Z})=\mathbb{Z}^{r}$. By the same argument $H_{1}(\mathcal{R}(f) ; G)=G^{r}$ for any coefficient group $G$, and dually $H^{1}(\mathcal{R}(f) ; R)=R^{r}$ for any coefficient ring $R$.

Proposition 5.1. The map $\pi \circ \iota: \Gamma(f) \rightarrow \mathcal{R}(f)$ induces isomorphisms 
- on fundamental groups groups

$$
(\pi \circ \iota)_{\#}=\pi_{\#} \circ \iota_{\#}: \mathbb{F}_{r} \rightarrow \mathbb{F}_{r},
$$

- on the first homology groups

$$
(\pi \circ \iota)_{*}=\pi_{*} \circ \iota_{*}: \mathbb{Z}^{r} \rightarrow \mathbb{Z}^{r},
$$

- and on the first cohomology groups

$$
(\pi \circ \iota)^{*}=\iota^{*} \circ \pi^{*}: R^{r} \rightarrow R^{r}
$$

for every coefficient ring $R$.

Corollary 5.2. $\quad$ i) If $\pi_{1}(M)$ is a finite group, (e.g. $M$ is simply connected) then the Reeb graph $\mathcal{R}(f)$ of every function is a tree.

ii) If $\pi_{1}(M)$ is an abelian group, or more general, a discrete amenable group, the Reeb graph $\mathcal{R}(f)$ of every function contains at most one loop.

Proof. If $\pi_{1}(M)$ is finite then $(\pi \circ \iota)_{\#}$ factorizes throughout a finite group, thus its image is a finite group and must not be an isomorphism.

Analogously, if $\pi_{1}(M)$ is abelian then $(\pi \circ \iota)_{\#}$ factorizes throughout an abelian group. Therefore its image is abelian and hence $(\pi \circ \iota)_{\#}$ can not be an isomorphism if $r \geqslant 2$. Since $\iota_{\#}$ is a monomorphism, $\pi_{1}(M)$ contains $\mathbb{F}_{r}$ for $r \geqslant 2$ as a subgroup. This is impossible if $\pi_{1}(M)$ is a discrete finitely generated amenable group (cf. $[10])$.

It is known that discrete nilpotent and solvable groups are amenable.

Corollary 5.3. Let $f: X \rightarrow \mathbb{R}$, be any $C^{1}$-function with isolated critical points.

- If $X=S^{n}$ for $n \geqslant 2$, or $X=\mathbb{C} P^{n}, \mathbb{R} P^{n}$ for any $n$, then $\mathcal{R}(f)$ is a tree.

- If $X=\mathbb{T}^{n}$ for any $n$ then $\mathcal{R}(f)$ is either a tree or is homotopy equivalent to a circle.

- If $X=M_{g}$ a closed orientable surface of genus $g$ then $\mathcal{R}(f)$ contains at most $2 g$ loops.

- If $X=M_{g}$ a closed non-orientable surface of genus $g$ then $\mathcal{R}(f)$ contains at most $g$ loops.

Proof. The first two points are a trivial application of Corollary 5.2. For the third point observe that the composition

$$
\mathbb{Z}^{r} \stackrel{\iota_{*}}{\longrightarrow} \mathbb{Z}^{2 g} \stackrel{\pi_{*}}{\longrightarrow} \mathbb{Z}^{r}
$$

is an isomorphism, thus we have $r \leqslant 2 g$. The same holds for the non-orientable case with $2 g$ replaced by $g$.

In the case when $f: M_{g} \rightarrow \mathbb{R}$ is a Morse function the number of loops in $\mathcal{R}(f)$ is equal to $g$ (see $[4$, Lemma $\mathrm{A}]$ ).

Now we strengthen our Proposition 5.3 showing that for any $C^{1}$-function $f$ on a closed orientable surface $M_{g}$ the number of loops in $\mathcal{R}(f)$ is lower or equal to the genus $g$ of $M$. We start with the following observation.

Lemma 5.4. Let $[p] \in \mathcal{R}(f)$ be a point which is in the interior of an edge, such that $\mathcal{R}(f) \backslash\{[p]\}$ is path-connected. Then $M \backslash \pi^{-1}([p])$ is path-connected. 
Proof. Let $\Gamma(f) \subset M$, be the one-dimensional complex as in Definition 4.4. Recall that the map $\left.\pi\right|_{\Gamma}: \Gamma(f) \rightarrow \mathcal{R}(f)$ contracts 'spanning trees' in essential components and is a homeomorphism elsewhere (see Proposition 4.5). Therefore the homotopy equivalence $\Gamma(f) \rightarrow \mathcal{R}(f)$ restricts to a homotopy equivalence

$$
\Gamma(f) \backslash \pi^{-1}([p]) \rightarrow \mathcal{R}(f) \backslash\{[p]\} .
$$

Since $\mathcal{R}(f) \backslash\{[p]\}$ is path-connected, $\Gamma(f) \backslash \pi^{-1}([p])$ is path-connected.

Take $x, y \in M \backslash \pi^{-1}([p])$. Let $x^{\prime}, y^{\prime} \in \Gamma(f) \subset M$ be two points such that $\pi(x)=\pi\left(x^{\prime}\right)$ and $\pi(y)=\pi\left(y^{\prime}\right)$. By Proposition 2.4 points $x$ and $x^{\prime}\left(y\right.$ and $\left.y^{\prime}\right)$ can be joined by a path $\alpha$ ( $\beta$, respectively) in the level set of $x$ (of $y$, respectively). Take a path $\gamma$ in $\Gamma(f) \backslash \pi^{-1}([p])$ connecting $x^{\prime}$ and $y^{\prime}$. Then $\alpha * \gamma * \beta^{-1}$ is a path in $M \backslash \pi^{-1}([p])$ joining $x$ and $y$.

Repeating the argument above several times we obtain the following corollary.

Corollary 5.5. Let $\left[p_{1}\right], \ldots,\left[p_{r}\right] \in \mathcal{R}(f)$ be points which are in the interior of edges. If $\mathcal{R}(f) \backslash\left\{\left[p_{1}\right], \ldots,\left[p_{r}\right]\right\}$ is connected, then $\left.M \backslash \pi^{-1}\left(\left\{\left[p_{1}\right], \ldots, p_{r}\right]\right\}\right)$ is connected.

Theorem 5.6. If $M_{g}$ is a closed orientable surface of genus $g$, then the number of loops in $\mathcal{R}(f)$ is less than or equal to $g$.

Proof. Let $L \subset \mathcal{R}(f)$ be a loop in the Reeb graph and $J \subset L$ an edge. If we remove from $\mathcal{R}(f)$ a point $[p] \in \int(J)$, then $\mathcal{R}(f) \backslash\{[p]\}$ is connected. Suppose now that $\mathcal{R}(f)$ has $r$ loops. Then

$$
\mathcal{R}(f) \backslash\left\{\left[p_{1}\right], \ldots,\left[p_{r}\right]\right\}
$$

is still connected, where each $p_{i}$ belongs to an edge in a unique loop. By Lemma 5.5

$$
M \backslash \pi^{-1}\left(\left\{\left[p_{1}\right], \ldots,\left[p_{r}\right]\right\}\right)
$$

is connected.

When $M_{g}$ is a compact surface, a connected component of the preimage of a regular point is a connected manifold of co-dimension 1, i.e. is homeomorphic to $S^{1}$. Thus after removing $r$ (disjoint) circles $M$ is still a connected space. This is possible only if $M_{g}$ has genus at least $r$.

The above theorem supplies a criteria to recognize a function which is not Morse.

On the other hand, most manifolds admit smooth functions with very few critical points. It is well known that on every oriented closed surface $M_{g}$ for $g \geqslant 1$ there exists a $C^{1}$-function $f: M_{g} \rightarrow \mathbb{R}$ with only three critical points (see [15, p. 90-91]). There are also manifolds of higher dimensions which admit $C^{1}$-functions with only three critical points. An example of such function, is the real or complex projective space of (projective) dimension 3 (see [8] for more information).

Theorem 5.7. Let $f: M \rightarrow \mathbb{R}$ be a $C^{1}$-function with only three critical points on a closed manifold. Then the Reeb graph $\mathcal{R}(f)$ is a tree with two edges.

We begin with the following lemma.

Lemma 5.8. Let $p \in M$ be a local extremum of a $C^{1}$-function $f: M \rightarrow \mathbb{R}$ with finite number of critical points. Then $[p] \in \mathcal{R}(f)$ is a vertex of degree 1. 
Proof. We assume that $p$ is a local maximum. The proof for a local minimum is analogous.

Suppose that $p \in M$ corresponds to a vertex of degree greater or equal than 2 and $f(p) \in(d, c)$. This implies that every sufficiently small neighbourhood $U$ of $p$ satisfies

$$
U \cap\left(M^{(d, c)} \backslash\{p\}\right)=X \sqcup Y,
$$

for some non-empty and disjoint sets $X$ and $Y$. Let $h: B \rightarrow \mathbb{R}^{n}$ be a chart around $p$ taking $p$ to 0 . If we take $U=B$ for a sufficiently small $B$ (i.e. $B \subset M^{(d, c)}$ ), the equality becomes

$$
B \backslash\{p\}=X \sqcup Y
$$

for some (possibly different, but still disjoint and non-empty) $X$ and $Y$. Then

$$
\mathbb{R}^{n} \backslash\{0\}=h(B \backslash\{p\})=h(X \sqcup Y)=h(X) \sqcup h(Y),
$$

which is clearly a contradiction since $\mathbb{R}^{n} \backslash\{0\}$ is connected for $n \geqslant 2$.

Proof of Theorem 5.7. Let $p^{+}, p^{-}, q$ denote respectively the maximum, the minimum, and third critical point of $f: M \rightarrow \mathbb{R}$. Let $f\left(p^{+}\right)=c^{\prime}, f\left(p^{-}\right)=c$, and $f(q)=d$ for some $c \leq d \leq c^{\prime}$. By Lemma 5.8 there exists a unique connected component $C^{+}\left(C^{-}\right)$of type (IIa) such that $p^{+} \subset \operatorname{cl}\left(C^{+}\right)\left(p^{-} \subset \operatorname{cl}\left(C^{-}\right)\right.$, respectively). Evidently $q \in \operatorname{cl}\left(C^{+}\right)$and $q \in \operatorname{cl}\left(C^{-}\right)$. Otherwise $q$ would be in an isolated component of a connected manifold $M$. Consequently $\mathcal{R}(f)$ consists of three vertices $\left[p^{+}\right],\left[p^{-}\right],[q]$, and two edges $J^{+}=\left[C^{+}\right]$, and $J^{-}=\left[C^{-}\right]$which proves the statement.

5.1. Final remarks. The results of this work show that the Reeb graph of a function $f: M \rightarrow \mathbb{R}$ is a not very fine combinatorial invariant of a the pair $(M, f)$. More information can be extracted from $\mathcal{R}(f)$ only if the fundamental group of $M$ contains a free group $\mathbb{F}_{r}$, on $r \geq 2$ generators. For example this works well for surfaces. This explains why the reverse representation of a surface constructed from the Reeb graph is attainable (see $[11,16]$ ). On the other hand in the case of surfaces one could expect to get more information by studying the embedding of $\Gamma(f)$ in $M$. In particular one can study the Bollobas-Riordan-Tutte polynomial of $\Gamma(f) \subset M$ and ribbon graphs.

It is a natural question what is the image of $F_{r}=\pi_{1}(\mathcal{R}(f))=\pi_{1}(\Gamma(f))$ in $\pi_{1}(M)$. A similar question has been answered in terms of horizontal homology by Dey and Wang in [5]. Furthermore, one can reverse the question and ask whether every subgroup of $\pi_{1}(M)$ isomorphic to $\mathbb{F}_{r}$ is in the image of $\pi_{1}(\mathcal{R}(f))$ for some function $f$.

Usually we want to simplify the manifold using $\mathcal{R}(f)$, so there is a natural tendency to seek for functions with the least possible number of critical points. However, when trying to infer some information about $M$ from its Reeb graph, we should try to make $\mathcal{R}(f)$ as complicated as possible, hence there is a need for construction of complicated functions on $M$ (with plenty of critical points and loops). To our knowledge there is no algorithm that provides such functions. A recent paper by Benedetti and Lutz [1] shows that even random Morse PL-functions tend to have a very simple Morse vectors. 


\section{REFERENCES}

[1] Benedetti, B. and Lutz F.H. Random Discrete Morse Theory and a New Library of Triangulations arXiv:1303.6422, 2013.

[2] Biasotti, S., Giorgi, D., Spagnuolo, M. and Falcidieno, B. Reeb graphs for shape analysis and applications, Theoret. Comput. Sci. 392 (2008), 5-22.

[3] Bollobás, B. and Riordan, O. A polynomial invariant of graphs on orientable surfaces, Proc. London Math. Soc. 83 (2001), no. 3, 513-531.

[4] Cole-McLaughlin, K., Edelsbrunner, H., Harer, J., Natarajan, V. and Pascucci, V. Loops in Reeb graphs of 2-manifolds, Discrete Comput. Geom. 32 (2004), 231-244.

[5] Dey, T. K. and Wang, Y. Reeb graphs: Approximation and persistence, Discr. \& Comput. Geom. 2013 Volume 49, Issue 1, pp 46-73.

[6] Katok, A. and Hasselblatt, B. Introduction to the Modern Theory of Dynamical Systems, Encyclopedia of Mathematics and its Applications, vol. 54, Cambridge University Press, 1995.

[7] Kronrod, A.S. On functions of two variables, Uspekhi Mat. Nauk 5 (1950), no. 1, 24-134.

[8] Milnor, J. Morse Theory. Princeton University Press, (1963).

[9] Nitecki, Z. Differentiable dynamics. An introduction to the orbit structure of diffeomorphisms, The M.I.T. Press, Cambridge, Mass.-London, 1971.

[10] Nowak, P.W. and Yu, G. Large scale geometry, European Mathematical Society (EMS), Zürich, 2012.

[11] Masumoto, Y. and Saeki, O. Smooth function on a manifold with given Reeb graph, Kyushu J. of Math. 65 no. 1, 75-84, (2011).

[12] Ore, O. Theory of graphs, Amer. Math. Soc. Colloq. Publ., Vol. 38, 1962.

[13] Prishlyak, A. Topological equivalence of smooth functions with isolated critical points on a closed surface, Topology Appl. 119 (2002), no. 3, 257-267.

[14] Reeb, G. Sur les points singuliers d'une forme de Pfaff complétement intégrable ou d'une fonction numerique, C. R. Acad. Sci. Paris 222, 847-849, (1946).

[15] Seifert, H. and Threlfall, W. Variationsrechnung its Grossen, (Theorie von Marston Morse), Leipzig and Berlin, Teubner, 1938. pp. 115

[16] Sharko, V.V. About Kronrod-Reeb Graph of a function on a manifold, Methods of Functional Analysis and Topology Vol. 12 (2006), no. 4, pp. 389-396.

Marek Kaluba: Adam Mickiewicz University in Poznań, Faculty of Mathematics and Computer Science, ul. Umultowska 87, 61-614 Poznań, Poland

E-mail address: kalmar@amu.edu.pl

Wacław Marzantowicz: Adam Mickiewicz University in Poznań, Faculty of Mathematics And Computer Science, ul. Umultowska 87, 61-614 Poznań, Poland

E-mail address: marzan@amu.edu.pl

Nelson Silva: Adam Mickiewicz University in Poznań, Faculty of Mathematics and Computer Science, ul. Umultowska 87, 61-614 Poznań, Poland

Current address: Institute of Mathematics and Computer Science, University of So Paulo, Avenida Trabalhador São-carlense, 400 - Centro, CEP: 13566-590, São Carlos, SP Brazil

E-mail address: nelson@icmc.usp.br 